\title{
Phase II trial of methylglyoxal bis-guanylhydrazone (MGBG) in refractory small cell lung cancer
}

\author{
Michael S. Simon, John Eckenrode, Ronald B. Natale \\ Department of Internal Medicine, Division of Hematology/Oncology, University of Michigan, \\ Ann Arbor, USA
}

Key words: MGBG, polyamine inhibitor, phase II study, small cell lung cancer

\section{Summary}

Methylglyoxal bis-guanylhydrazone (MGBG), a potent inhibitor of polyamine synthesis, has demonstrated single agent activity against a number of tumor types including malignant lymphomas and head and neck, esophageal and non-small cell lung cancers. The growth of small cell lung cancer (SCLC) cell lines can be arrested by polyamine inhibition. Therefore a phase II trial was conducted in twenty-four patients with refractory SCLC. MGBG was administered by intravenous infusion at a dose of $500 \mathrm{mg} / \mathrm{m}^{2}$ per week for four cycles and then every two weeks thereafter. The dose was escalated by $100 \mathrm{mg} / \mathrm{m}^{2}$ every two weeks in the absence of toxicity $\geq$ grade 2 . One patient achieved a partial response of objectively measurable lung disease and supraclavicular adenopathy. Three patients had stable disease. Dose limiting toxicity consisted primarily of mild to moderate nausea, vomiting, stomatitis and/or diarrhea. Myelosuppression was uncommon and rarely dose limiting. We conclude that MGBG in the dose and schedule used does not have significant activity as a single agent in previously treated small cell lung cancer.

\section{Introduction}

Methylglyoxal bis-guanylhydrazone (MGBG) is a synthetic polycarbonyl compound that has been shown to have useful antitumor activity in patients with advanced malignant lymphomas and head and neck, esophageal and non-small cell lung cancers [1]. Although the exact mechanism of its antitumor activity is not known, MGBG produces a variety of metabolic effects including mitochondrial swelling, uncoupling of oxidative phosphorylation and polyamine depletion [2]. Through potent inhibition of S-adenosylmethionine decarboxylase (SAM-DC), MGBG blocks formation of the amino propyl donor required for the synthesis of spermine and spermidine from putrescine in the polyamine pathway [3]. Inhibition of polyamine biosynthesis is of interest in that high and unperturbed polyamine production appears to be essential to the growth of several human tumors including small cell lung cancer (SCLC) [4-6]. For these reasons we chose to conduct a phase II trial of MGBG in highly selected patients with refractory SCLC. The following report details the methods and results of the study.

\section{Materials and methods}

Twenty-four patients with objectively measurable and histologically confirmed SCLC were entered in the study. Eligibility requirements included Karnofsky performance score $\geq 50$, white blood cell (WBC) count $\geq 4000 / \mathrm{mm}^{3}$, platelet count $\geq$ $100,000 / \mathrm{mm}^{3}$ blood urea nitrogen $\leq 20 \mathrm{mg} \%$, serum creatinine $\leq 1.6 \mathrm{mg} \%$, serum bilirubin $\leq$ $2.0 \mathrm{mg} \%$, no treatment for at least the preceding 
4 weeks and informed consent. At the start of therapy all patients had a complete medical history, physical examination, and laboratory evaluation including complete blood cell (CBC) count with differential and platelet count, fasting blood glucose, urinalysis, 12-channel biochemical screening profile, carcinoembryonic antigen (CEA) and chest roentgenogram. Radionuclide bone and liver scans and chest/abdominal CT scans were obtained as needed. Each patient had a weekly physical examination, toxicity evaluation, $\mathrm{CBC}$, and serum chemistries and a monthly evaluation of chest roentgenogram or diagnostic scan as needed for tumor measurements.

MGBG was administered by injection through an established free-flowing iv line over 30 minutes. The initial dose of $500 \mathrm{mg} / \mathrm{m}^{2}$ was administered weekly for four cycles and then on alternate weeks. Criteria used for dose adjustments followed the standard toxicity criteria. The dose was escalated by $100 \mathrm{mg} / \mathrm{m}^{2}$ in the absence of toxicity $\geq$ grade 2 . The dose was attenuated by $25 \%$ in the presence of grade 3 toxicity and $50 \%$ in the presence of grade 4 toxicity. An adequate trial required a minimum of three cycles of MGBG.

Therapeutic response criteria were defined as: partial response (PR) $-\geq 50 \%$ decrease of all measurable lesions for at least 4 weeks: Disease progression (PD) $-\mathrm{a} \geq 25 \%$ increase in the sum of the products of measureable lesions or the appearance of new lesions; and stable disease (STAB) disease regression less than that needed for PR or disease progression less than that needed for PD for at least two months.

\section{Patient characteristics}

There were 15 males and 9 females with a median age of 59 years (range, 47-71) and a median Karnofsky performance status of 70 (range, 50-90). All patients had received prior combination chemotherapy including cyclophosphamide $(100 \%)$, doxorubicin (96\%), vincristine (96\%), VP-16-213 $(46 \%)$, cisplatin $(46 \%)$, methotrexate $(22 \%)$, CCNU $(22 \%)$, or other $(25 \%)$. Sixteen patients $(67 \%)$ had received only one prior combination chemotherapy treatment regimen. Ten patients $(\mathbf{4 2 \%})$ had received radiation therapy to whole brain and eight patients $(33 \%)$ had received it to the mediastinum. Sixteen patients $(67 \%)$ had more than one site of tumor involvement at entry into this study including lung (2), lymph nodes (6), liver (7), spleen (1), adrenal (2), bone (2), and subcutaneous tissue (1).

\section{Results}

Twenty-two of 24 patients entered into the study are considered evaluable for response and toxicity. The two unevaluable cases include one patient who had an unrelated death 5 days after the first dose of MGBG and another who was lost to follow up after 2 doses. There was only one objective response (PR) in 22 evaluable patients (observed response rate $=4.5 \%, 95 \%$ confidence interval $=0.2-$ $19.8 \%$ ). The response occurred after 2 doses of MGBG in objectively measurable lung disease and supraclavicular adenopathy in a patient whose disease had progressed after a partial response to CAV chemotherapy. The patient died after 6 cycles $(2$ months) of MGBG due to progressive brain metastases but remained in PR extracranially. Three additional patients had stable disease.

The most common and dose limiting toxicities occurred in the gastrointestinal tract. Mild to moderate (grade 1-2) diarrhea and/or stomatitis occurred in 10 patients $(45 \%)$, nausea and vomiting occurred in 10 patients $(45 \%)$ and anorexia occurred in 7 patients $(32 \%)$. Grade $1-3$ myalgias and/or fatigue occurred in 8 patients $(36 \%)$. Leukopenia and thrombocytopenia were uncommon and rarely dose limiting. Only two patients developed grade 1 leukopenia, and thrombocytopenia occurred in 5 patients $(3=$ grade $1,1=$ grade 2 , and $1=$ grade 4 ). One patient complained of moderate to severe jaw pain for 1-2 days after each dose of MGBG and one patient developed grade 1 dermatitis.

The median number of doses of MGBG given was 4 per patient (range, 1-8). Two patients required dose attenuations due to myalgias, thrombocytopenia or gastrointestinal toxicity and three 
patients tolerated dose escalations (one to 800 $\mathrm{mg} / \mathrm{m}^{2}$ with only grade 1 toxicity).

\section{Discussion}

The resurgence of interest in MGBG in the late 1970's was due to the development of a more tolerable weekly schedule of administration $[1,7]$ and the discovery of its potency as an polyamine inhibitor [3]. The products of polyamine synthesis, putrescine, spermidine and spermine have been implicated in the maintenance of cell growth and it was hypothesized that the inhibition of this pathway could provide an effective tool for arresting the growth of cancer cells $[4,5]$. Alpha-difluoromethylornithine (DFMO), an irreversible inhibitor of ornithine decarboxylase which catalyzes the rate limiting step in polyamine synthesis, has been of particular interest. Luk et al. [6] have shown that the growth and viability of human SCLC cells in culture is markedly inhibited by DFMO. However, only one partial response has been observed in 33 SCLC patients treated with DFMO in phase I and II trials [8-10].

In this study, MGBG demonstrated little activity (one PR) in 24 good performance status patients with SCLC, 16 of whom had received only one prior treatment regimen. Although its pattern of toxicity, including lack of significant myelosuppression, make $\mathrm{MGBG}$ an attractive agent to incorporate into combination regimens used in SCLC, this study as well as the negative one reported by Scher et $a l$. which used a dose and schedule similar to ours [11] fail to justify further studies of this compound.

\section{Acknowledgements}

The authors wish to thank Ms. Donna Baisden for her help in preparing this manuscript. This work was supported in part by contract NO1-CM-07 405, Division of Cancer Treatment, National Cancer In- stitute, Department of Health and Human Services, Bethesda, Maryland.

\section{References}

1. Warrell RP, Burchenal JH: Methylglyoxal-bis (guanylhydrazone) (methyl-GAG): Current status and future prospects. J Clin Oncol 1: 52-65, 1983

2. Pine MJ, Dipaolo JA: The antimitochondrial action of 2-chloro-4',4' '-bis (2-imidazolin-2-yl) terephthalanilide and methyl-glyoxal bis (guanyl hydrazone). Cancer Res 26: $18-25,1966$

3. Williams-Ashman HG, Scheone A: Methylglyoxal bis(guanyl hydrazone) as a potent inhibitor of mammalian and yeast S-adenosylmethionine decarboxylases. Biochem Biophys Res Commun 46: 288-295, 1972

4. Pegg AE, McCann PP: Polyamine metabolism and function. Am J Physiol 243: C212-C221, 1982

5. Russell DH: Increased polyamine concentrations in the urine of human cancer patients. Nature (New Biol) 233: $144-145,1971$

6. Luk GD, Goodwin G, Marton LJ, Baylin SB: Polyamines are necessary for the survival of human small-cell lung carcinoma in culture. Proc Natl Acad Sci USA 78: 2355-2358, 1981

7. Knight WA, Livingstone RB, Fabian C, Constanzi J: Phase I-II trial of methyl-GAG: a southwest oncology group pilot study. Cancer Treat Rep 63: 1933-1937, 1979

8. Abeloff MD, Slavik M, Luk GD, Griffin CA, Hermann J, Blanc O, Sjoerdsma A, Baylin SB: Phase I trial and pharmacokinetic studies of alpha-difluoromethylornithine - an inhibitor of polyamine biosynthesis. J Clin Oncol 2: 124-130, 1984

9. Abeloff MD, Rosen ST, Luk GD, Baylin SB, Zeltzman M, Sjoerdsma A: Phase II Trials of alpha-difluromethylornithine, an inhibitor of polyamine synthesis, in advanced small cell lung cancer and colon cancer. Cancer Treat Rep 70: $843-845,1986$

10. Griffin CA, Slavik M, Chien SC, Hermann J, Thompson G, Blanc O, Luk GD, Baylin SB, Abeloff MD: Phase I trial and pharmacokinetic study of intravenous and oral alpha-difluoromethylornithine. Invest New Drugs 5: 177-186, 1987

11. Scher H, Chapman R, Kelsen D, Gralla R, Wittes R: Phase II trial of mitoguazone in patients with relapsed small cell carcinoma of the lung. Cancer Treat Rep 68: 561-562, 1984

Address for offprints: R.B. Natale, University of Michigan, Upjohn Center, Box 0504, 1310 E. Catherine Drive, Ann Arbor, MI 48109, USA 